\title{
Generation of fine fragments during dynamic propagation of pressurized cracks
}

\author{
A. Iravani $\odot,{ }^{1}$ F. Ouchterlony $\odot,{ }^{1}$ I. Kukolj, ${ }^{1}$ and J. A. Åström ${ }^{2}$ \\ ${ }^{1}$ Department of Mineral Resources Engineering, Montanuniversität Leoben, A8700 Leoben, Austria \\ ${ }^{2}$ CSC-IT Center for Science, P.O.Box 405, FIN-02101 Esbo, Finland
}

(Received 26 March 2019; revised manuscript received 13 November 2019; accepted 24 December 2019; published 24 February 2020)

\begin{abstract}
High-resolution numerical simulations of cracks driven by an internal pressure in a heterogeneous and brittle granular medium produce fragment-size distributions with the same characteristics as experiments on blasted cylinders of mortar and rock in both the fine- and the intermediate-size-fragment regions. To mimic full-scale blasts used, e.g., within the mining industry, the cracks propagate in a medium that is under compression, neutral, or under tension. In a compressive environment, shear fracture produces a large volume of fines, whereas in a neutral or tensile environment, unstable crack branching is responsible for a much smaller volume of fines. The boundary between the fine- and the intermediate-size fragments scales as the average grain size of the material. The ultimate goal is to develop a blasting process that minimizes the fines, which, in mining, are both an environmental hazard and useless for further processing.
\end{abstract}

DOI: 10.1103/PhysRevE.101.023002

\section{INTRODUCTION}

A fundamental process within the mining industry is the blasting of rock to extract minerals. This process creates a lot of dust that cannot be processed further and that is also an environmental hazard. It would, therefore, be of great significance to minimize the dust which is often called "fines." The blasting process is carried out by drilling cylindrical holes and blasting them from within. The blast creates a conical compressive shock wave that travels outward in the radial direction of the cylinder. As the hole expands outward, it will induce tension in the tangential direction. The stress waves emanating from the borehole will sooner or later reach a boundary or an interface between two dissimilar materials. Here, the specific acoustic impedance of the host rock plus the angle of wave incidence define the stress waves interaction at the boundary, such as mode transformation, transmission, reflection, and refraction [1]. Reflected waves often meet cracks initiated by the primary wave. In this way, propagating cracks may experience both compressive and tensile environments that influence the fines production.

Well-known theories within mining engineering for blastinduced fines typically postulate compressive and/or shear failure as the main mechanism for fine-fragments production, such as the crushed zone model (CZM) [2], the two component model [3], and the additions to CZM by Onederra et al. [4]. However, when blasting cylinders of a given size with a decreasing amount of explosives, a critical charge size is eventually reached. At this point, the cylinder barely falls

Published by the American Physical Society under the terms of the Creative Commons Attribution 4.0 International license. Further distribution of this work must maintain attribution to the author(s) and the published article's title, journal citation, and DOI. apart into a few large blocks plus a small volume of very fine fragments $[5,6]$. In such a case, a crushed zone around the borehole and crushing due to fragment collisions normally do not occur, and the theories fail. An inspection of the outcome of such experiments reveals that the fines originate along the propagation paths of the cracks that create the large blocks. This indicates that the key to understanding the blast-induced fines production can be found in the detailed modeling of cracks driven by the internal pressure induced by the blast and propagating in a medium that is under externally applied stresses that can be either compressive or tensile.

Within the physics community, fragmentation research has typically been concentrated around the possible universality of scale-invariant power-law fragment size distributions (FSDs). There seems to be limited universality, but there are dependencies on dimensions, and compressive or impact fragmentation seems to behave differently than tensile fragmentation [7-11].

In this article, we report the results of blasting experiments and numerical simulations of propagating cracks driven by internal pressure and propagating under specific external stress states that mimic realistic conditions for full-scale blasting. The model materials are granular both in the simulations and in the experiments with an average granularity on the order of $0.1 \mathrm{~mm}$. The main difference between the experiments and the simulations is that, in the simulations, the grains are indestructible, whereas in the experiments, the grains can fragment, and there is also an intergranular matrix that can form fragments much smaller than the typical grain size. Nevertheless, we are able to demonstrate that the FSDs for experiments and simulations are very similar and that they can be understood in terms of universal processes.

Here, we find that the granularity length scale determines a boundary between fine- and intermediate-size fragments. In the intermediate-size region, the FSD has the universal form of the crack branching-merging process, whereas in the fines 
region, the FSD has the form induced by shear crushing if the external stresses are compressive and a branching-merging process if the external stresses are tensile or neutral. In the latter case, the boundary between the two regions appears as an offset in the FSD. As a consequence, we find that the total volume of fines in blasting is affected by the granularity of the material that is blasted, the stress environment of the propagating cracks, and the total charge used in the blast.

\section{FRAGMENTATION THEORY}

The magnitude of the external compressive stresses surrounding a pressurized crack has direct influence on its speed of propagation. For example, a high compressive stress that acts perpendicular to the crack flanks reduces the propagating velocity of the crack tip. Depending on the magnitude of the compressive stress and the crack's driving force, the velocity may eventually drop below the critical branching velocity of the material $V_{c}$ [12]. This process may lead to crack closure and at even higher compressive stresses cause crushing by compressive shear. In compressive shear, the debris will be crushed into decreasingly smaller fragments by grinding and compaction. This process may perhaps be conceptualized as a hierarchical process in which ever smaller fragments are broken to fill pore space that opens up in continuous shear deformation [13,14], or it may have similarities with impact fragmentation [10,11]. In both cases, the FSD can be approximated by a power-law fragment size distribution of the form $n_{\text {crush }}(s) d s \propto s^{-\beta} d s$ where the exponent $\beta$ depends on the material and the specific way it is fragmented and may take values roughly in the range of 1.8-3.5 [7,11]. Dimensionless size $s$ is here measured in the number of grains in fragments of a granular material.

In the absence of externally applied compressive stresses suppressing the propagating crack, the velocity of the crack propagation increases to a value close to $V_{c}$ where the crack begins to branch [12]. Crack branches readily merge to form fines as can be seen in Fig. 3. This branching-merging process is inherently universal and leads to a characteristic FSD [7,15]. This can be written as $n_{b m}(s) d s \propto s^{-\alpha} \exp \left(-s / s_{i}\right) d s$ with $\alpha=(2 D-1) / D$, where $D$ is the dimension (i.e., $D=$ 2,3 for membranes and bulk objects, respectively) $[7,15,16]$. The branches propagate for a short distance and are arrested in the stress free wake region created by the main propagating crack [12] inducing a size cutoff defined by $s_{i}$. The size cutoff is here approximated by an exponential function [15].

On the scale of the grain size in a granular material, we expect that the processes for fines formation described above are highly influenced by the granularity of the material. On larger scales, we expect the material to behave, such as a continuous material. It is, thus, reasonable to postulate that, in a granular material, there needs to be separate terms in the FSD for fragments at and below the grain sizes $s_{f}$ and for larger intermediate-size fragments $s_{i}$. For the blasting scenarios investigated here, we expect that the fines are created by either crushing or crack branching merging, whereas the intermediate-size fragments appear as a result of crack branching merging only. In principle, there could obviously be crushing also at the intermediate-size scale, but within the context investigated here, this does not happen.
The largest fragments, often called boulders, are the ones delineated by the main cracks. The main cracks can typically be assumed to propagate independent of each other and thereby to form a Poisson process [17], which induces an exponential term at the largest sizes of the FSD $n_{b}(s) d s \propto$ $\exp \left(-s / s_{b}\right) d s$, where $s_{b}$ sets the characteristic size of the boulders.

This would give the following options for the FSDs with right-hand side terms describing fines, intermediate-size fragments, and boulders, respectively:

$$
\begin{aligned}
n(s) d s= & C_{1} s^{-\beta} d s+C_{2} s^{-\alpha} \exp \left(-\frac{s}{s_{i}}\right) d s \\
& +C_{3} \exp \left(-\frac{s}{s_{b}}\right) d s,
\end{aligned}
$$

which we would expect to appear for blasting under externally applied compressive stress, whereas, in a tensile environment, we would expect

$$
\begin{aligned}
n(s) d s= & C_{1} s^{-\alpha} \exp \left(-\frac{s}{s_{f}}\right) d s+C_{2} s^{-\alpha} \exp \left(-\frac{s}{s_{i}}\right) \\
& +C_{3} \exp \left(-\frac{s}{s_{b}}\right) d s,
\end{aligned}
$$

where $C_{1}, C_{2}$, and $C_{3}$ are constants that determine the relative weights of the different fragment-size regions.

A good way to estimate the validity of Eqs. (1) and (2) is to relate them to the mass passing fraction $[\operatorname{MPF}(x)]$, that is, the common function used to characterize fragmentation results within the mining community [18]. The $\operatorname{MPF}(x)$ is defined as the fraction of mass for a collection of fragments that passes through a successive set of sieves with decreasing mesh sizes $x$. That is, for a mesh size of $x=0, \operatorname{MPF}(x)=0.0$, and $\operatorname{MPF}\left(x>x_{\max }\right)=1.0$ or $100 \%$, where $x_{\max }$ is the largest fragment.

With the transformation $s \approx\left(x / x_{\text {grain }}\right)^{3}, d s=3 x^{2} / x_{\text {grain }}^{3} d x$ for $D=3$, the $\operatorname{MPF}(x)$ can be written

$$
\operatorname{MPF}(x)=\frac{m_{\text {grain }}}{M_{0}} \frac{3}{x_{\text {grain }}^{3}} \int_{x_{\text {grain }}}^{x} x^{\prime 3} n\left(s\left(x^{\prime}\right)\right) x^{\prime 2} d x^{\prime},
$$

where $M_{0}$ is the total mass, $x_{\text {grain }}$ is the grain size of the material, and $m_{\text {grain }}$ the mass of a grain. This equation can be written as a sum of two incomplete $\Gamma$ functions. It is, however, more useful to examine Eq. (3) in the case where $n_{b}(s)$ is neglected and the exponential parts of the first two terms in Eqs. (1) and (2) are replaced by limited ranges of integration. That is, the first term is assumed to be valid within the interval $\left[x_{\text {grain }}, x_{\text {trans }}\right]$, and the second term is assumed to be valid within the interval $\left[x_{\text {trans }}, x_{\max }\right]$, where $x_{\text {trans }} \propto s_{f}^{1 / 3}$ is the transition size between fines- and intermediate-size fragments [cf. Fig. 1(b)], and $x_{\max } \propto s_{b}^{1 / 3}$ is the maximum size of boulders. If we neglect the term on the order of $x_{\text {grain }} / x_{\max }$, we get for the $\operatorname{MPF}(x)$,

$$
\operatorname{MPF}(x)=f_{f p}\left[\left(\frac{x}{x_{\text {grain }}}\right)^{-3\langle\alpha, \beta\rangle+6}-1.0\right]+f_{i p}\left(\frac{x}{x_{\text {max }}}\right)^{-3 \alpha+6},
$$


(a)

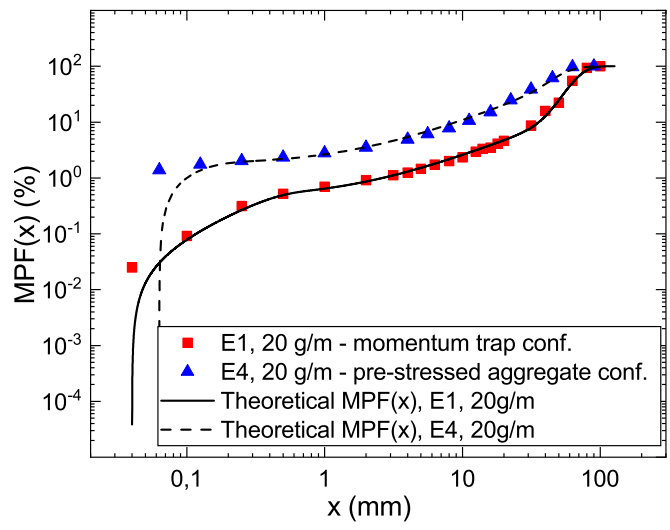

(b)

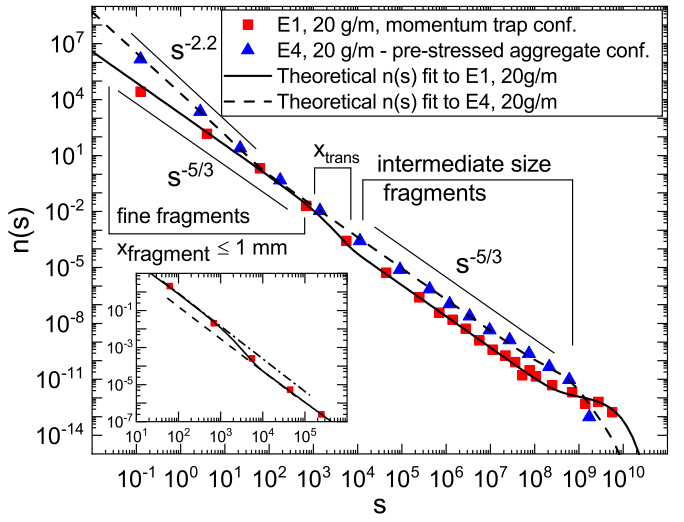

FIG. 1. (a) Two sets of experimental MPFs: The red squares and blue triangles, and the two corresponding theoretical MPF $(x)$ fits: The solid and the dashed black lines. The data correspond to two blasted cylinders confined with MT (E1) [21,22] and prestressed (E4) [6] boundary conditions, respectively. (b) The corresponding $n(s)$ for the same data together with fits to Eqs. (1) and (2). Here, the first data point on the $s$ axis refers to the smallest detectable fragment.

where $\langle\alpha, \beta\rangle$ means either $\alpha$ or $\beta$ depending on which $n(s)$ equation, i.e., Eqs. (1) and (2), is used. The factors $f_{f p}$ and $f_{i p}$ are constants,

$$
\begin{gathered}
f_{f p}=\frac{m_{\text {grain }}}{M_{0}} \frac{3 C_{1}}{-3\langle\alpha, \beta\rangle+6}, \\
f_{i p}=\frac{m_{\text {grain }}}{M_{0}} \frac{3 C_{2} x_{\max }^{-3 \alpha+6}}{\left(x_{\text {grain }}^{-3 \alpha+6}\right)(-3 \alpha+6)} .
\end{gathered}
$$

For $D=3$, the value of $-3 \alpha+6$ is equal to 1 , and, thus, the second part on the right-hand side of Eq. (4) becomes the empirical Gates-Gaudin-Schuhmann distribution (GGS) [19] which is a power-law function with an exponent $m=1$, and the first part represents the characteristic shape of the MPFs for the fine fragments. This is consistent with the typical functional forms observed for the MPFs: a nonuniversal shape for the fines, followed by a wider or a narrower interval described by the GGS function, and finally a nonuniversal shape for the boulders. Representative examples are displayed in Fig. 1(a). A method of the reverse transformation from $\operatorname{MPF}(x)$ to $n(s)$ is given in the Appendix.

\section{EXPERIMENTS}

\section{A. Experimental setup}

We perform a set of blasting experiments, and together with data from earlier experiments, we have fragmentation data for 28 controlled blasting experiments of cylinders of sandstone [20], granite, mortar, and magnetic mortar (MM) [6]. The cylinders are blasted from within a central borehole along the axis of the cylinder. There are three different outer boundary conditions: (1) At the outer boundary of the cylinders, there is a layer of aggregate under $1 \mathrm{MPa}$ pressure, a so-called prestressed aggregate [6], (2) a momentum trap (MT) $[21,22]$ which, at the outer boundary, absorbs much of the incident compressive wave induced by the blast, and (3) an "unconfined" or a free outer boundary at which the initial compressive wave is reflected as a tensile wave. The logic behind studying these different outer boundary conditions is to model different blasting scenarios with the cracks forming in tensile, neutral, and compressive environments.

Details of the experiments are listed in Table I. The table is sorted by the sieving technique. Initially, the postmortem

\begin{tabular}{|c|c|c|c|c|c|}
\hline No. & Material & No. of blasted cylinders & $\begin{array}{l}\text { Charge density/specific charge } \\
(\mathrm{g} / \mathrm{m}) /\left(\mathrm{kg} / \mathrm{m}^{3}\right)\end{array}$ & Confinement & $\begin{array}{l}\text { Minimum mesh size } \\
(\mathrm{mm})\end{array}$ \\
\hline \multicolumn{6}{|c|}{ Mechanical sieving } \\
\hline E1 & Mortar & 3 & $6,12,20$ & MT & 0.04 \\
\hline $\mathrm{E} 2$ & Granite & 3 & $6,12,20$ & MT & 0.04 \\
\hline E3 & MM [6] & 5 & $3,5,10,20,40$ & Unconfined & 0.063 \\
\hline $\mathrm{E} 4$ & MM [6] & 4 & $5,10,20,40$ & Prestressed aggregate & 0.063 \\
\hline \multicolumn{6}{|c|}{+ sedimentation } \\
\hline$\overline{\mathrm{E} 5}$ & Sandstone $[20]$ & 6 & $0.46,0.444,0.431,0.231,0.218,0.22$ & Unconfined & 0.002 \\
\hline \multicolumn{6}{|c|}{ +laser diffraction } \\
\hline E6 & Mortar & 3 & $6,12,20$ & MT & 0.0004 \\
\hline E7 & Granite & 4 & $6,12,20$ & MT & 0.0004 \\
\hline
\end{tabular}

TABLE I. Experimental configurations. 
(a)

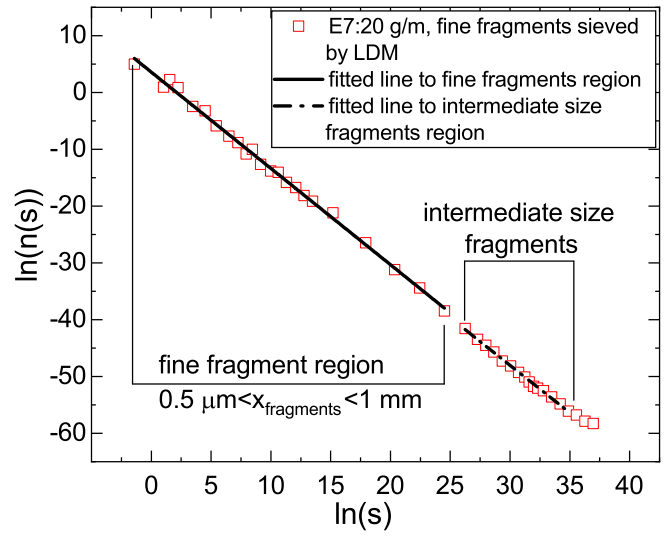

(b)

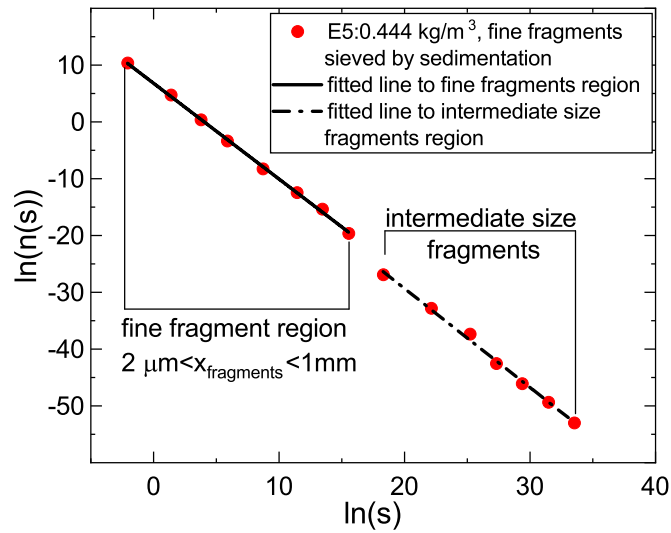

FIG. 2. Two fitted lines of the fine-fragments region and intermediate-size-fragment region shown as the solid and dashed lines, respectively. In (a), the data points (the square symbols) correspond to experiment E7 with $20 \mathrm{~g} / \mathrm{m}$ of PETN, and, in (b), the data points (the filled circles) correspond to experiment E5 with $0.444 \mathrm{~kg} / \mathrm{m}^{3}$, see Table I. The regression function and parameters of these fits are listed in Table II.

cylinders are mechanically sieved with minimum mesh sizes of either 0.063 or $0.04 \mathrm{~mm}$ to a maximum fragment size until a full MPF curve is formed. The fine fragments of the experiments E1 and E2 were apart from mechanical sieving also measured using a laser diffraction method (LDM) with a resolution down to $0.4 \mu \mathrm{m}$. The results from the LDM were then merged with the mechanical sieving data, thus, forming a wider range FSD. These are listed as E6 and E7. The fine fragments of the experimental tests listed in E5 were also additionally measured using a sedimentation method [20].

\section{B. Experimental results}

A prestressed and a momentum trap $\operatorname{MPF}(x)$ are displayed in Fig. 1(a) together with fits to Eq. (3). The theoretical $\operatorname{MPF}(x)$ functions are obtained by numerical integration and with a constant material grain size $x_{\text {grain }}$. For real materials, $x_{\text {grain }}$ is obviously not a constant (cf. Fig. 3). The $x_{\text {grain }}$ values used are listed in the last column in Table I as the "Minimum mesh size." The deviation between the experimental and the theoretical MPFs for the smallest fragments is a result of the theory assuming a minimum fragment size of a single grain, whereas the experimental data lump all masses smaller than the resolution into a single point.

The same data in the $n(s)$ form is displayed in Fig. 1(b). The differences between pre-stressed and MT boundary conditions become evident when presented in this form: (i) In the fine-fragments region of the prestressed cylinder, the FSD has a much steeper slope with a power-exponent $\beta \approx 2.2$ as indicated in the figure. For the cylinder confined by MT, the exponent is the expected $\alpha \approx 5 / 3$. (ii) At a fragment size of $s \sim 10^{2}$ for the prestressed case, there is a crossover to the branching-merging power-law $\alpha \approx 5 / 3$, whereas for the MT case, there appears the same power exponent for both finesand intermediate-fragment sizes, but there is an offset to lower fragment number by about an order of magnitude at $s \sim 10^{3}$. (iii) There are crossovers to exponential FSDs in both cases at the boulder size. There is a more pronounced boulder part for the MT case, and the boulders are slightly bigger. In the intermediate-fragment-size region, i.e., $x_{\text {trans }}<x<x_{\text {boulders }}$, the power exponents are identical $\alpha \approx 5 / 3$, but there are more intermediate-size fragments for the prestressed case.

With these results, it becomes evident that the more interesting case from a blasting technology point of view is the MT boundary condition as it produces significantly less fines than the prestressed case.

In order to test the statistical significance of the offset in the FSD of the cylinders confined by MT, we perform a statistical analysis to determine whether the slopes (gradients) of the two fitted power laws for the fine fragments and the intermediate-size fragments are significantly different, and whether the offset between these lines is significant [23].

For the analysis, we first use two sets of data: (1) the wider range FSD obtained by the LDM for granite (E7) blasted with $20 \mathrm{~g} / \mathrm{m}$ of pentaerythritol tetranitrate (PETN) with 23 data points forming the fine-fragments region, and (2) the data from sedimentation of fine fragments of sandstone (E5) blasted with $0.444 \mathrm{~kg} / \mathrm{m}^{3}$ with eight data points forming the fine-fragments region. The reason for the choice of these two datasets is to lessen the inherent systematic error of each sieving technique on the resultant FSDs. Here, for the analysis, the data points between the two lines, if any, and the ones forming the boulder part are not considered, see Figs. 2(a) and 2(b). The results of the linear regression are given in Table II.

The results of the statistical analysis for the significance of the two fitted power-law lines are given in Table III. The two large correlation coefficients $(|R| \approx 1)$ and their highly significant $p$ values, i.e., $p(R)$, indicate the existence of the two legitimate lines to compare. The $p$ value of the residual variance for experiment (E7) illustrates a significant difference between the two variances. Napier-Munn [23] suggests considering smaller value of $p(F)$ for gradient and intercept tests. The comparison of the gradients with $p(F)=0.202$ emphasizes that the two lines are parallel, i.e., the difference in the slope values is statistically insignificant. The small $p$ value of the intercept suggests that a real separation exists between the two parallel lines.

We analyzed all available data sets except those with the prestressed boundary condition in the same fashion as above. 
TABLE II. Regression analysis, E7: $20 \mathrm{~g} / \mathrm{m}$ and E5: $0.444 \mathrm{~kg} / \mathrm{m}^{3}$.

\begin{tabular}{|c|c|c|}
\hline Fitting region & Fine-fragments region & Intermediate-size-fragments region \\
\hline \multicolumn{3}{|c|}{ Equation } \\
\hline Parameter & $\ln \left(C_{1}\right)+\alpha \ln (s)$ & $\ln \left(C_{3}\right)+\alpha \ln (s)$ \\
\hline \multicolumn{3}{|l|}{ E7 } \\
\hline $\ln \left(C_{1}\right)$ & $3.59 \pm 0.25$ & \\
\hline $\ln \left(C_{3}\right)$ & & $2.04 \pm 0.69$ \\
\hline$\alpha$ & $-1.695 \pm 0.021$ & $-1.669 \pm 0.022$ \\
\hline$R^{2}$ & 0.9969 & 0.9977 \\
\hline Adjusted $R^{2}$ & 0.9967 & 0.9975 \\
\hline \multicolumn{3}{|l|}{ E5 } \\
\hline $\ln \left(C_{1}\right)$ & $6.80 \pm 0.19$ & \\
\hline $\ln \left(C_{3}\right)$ & & $5.49 \pm 1.24$ \\
\hline$\alpha$ & $-1.687 \pm 0.020$ & $-1.742 \pm 0.045$ \\
\hline$R^{2}$ & 0.9991 & 0.9966 \\
\hline Adjusted $R^{2}$ & 0.9990 & 0.9959 \\
\hline
\end{tabular}

In all 24 data sets, a significant offset between the fine- and the intermediate-size fragments' fitted lines exist, and, in 22 of them, the fine- and intermediate-size fragment's fitted lines are parallel. The two data sets that have different gradients are E5: $0.46 \mathrm{~kg} / \mathrm{m}^{3}$ and E5: $0.431 \mathrm{~kg} / \mathrm{m}^{3}$. The exponent of the fine-fragment-size distribution for both these sets is $\alpha \approx 5 / 3$, but the intermediate-size one deviates slightly in these two cases. Consequently, with all data considered, the experimental MPFs can be described by Eq. (2) unless the prestressed boundary condition is applied.

The postmortem fractures and crack flanks of the blasted cylinders E1 and E2, see Table I, have been analyzed by a scanning electron microscope (SEM). Figure 3 shows two images of crack branching and merging in granite (E2: $20 \mathrm{~g} / \mathrm{m})$ and mortar $(\mathrm{E} 1: 12 \mathrm{~g} / \mathrm{m})$ both with similar fields of

TABLE III. Statistical significance study of the fines- and intermediate-size-fragments' fitted lines, E7: $20 \mathrm{~g} / \mathrm{m}$ and E5: $0.444 \mathrm{~kg} / \mathrm{m}^{3}$.

\begin{tabular}{lcc}
\hline \hline Test & $\begin{array}{c}\text { Fine-fragments } \\
\text { region }\end{array}$ & $\begin{array}{c}\text { Intermediate-size- } \\
\text { fragments region }\end{array}$ \\
\hline $\mathrm{E} 7$ & & \\
\hline No. of data points & 23 & 17 \\
$R$ & -0.998 & -0.998 \\
$p(R)$ & $8.42 \times 10^{-28}$ & $1.09 \times 10^{-20}$ \\
Residual variance & $F=6.818$ & $p(F)=0.0002$ \\
Gradient & $F=1.691$ & $p(F)=0.202$ \\
Intercept & $F=4.556$ & $p(F)=0.039$ \\
E5 & & \\
\hline No. of data points & 8 & 7 \\
$R$ & -0.999 & -0.998 \\
$p(R)$ & $2.03 \times 10^{-10}$ & $2.28 \times 10^{-7}$ \\
Residual variance & $F=3.277$ & $p(F)=0.090$ \\
Gradient & $F=1.439$ & $p(F)=0.255$ \\
Intercept & $F=21.45$ & $p(F)=0.0005$ \\
\hline \hline
\end{tabular}

view of $0.179 \mathrm{~mm} \times 0.785 \mathrm{~mm}$. Figure 3(a) shows two radial cracks (radial in the sense of propagating in an outwards radial direction of the cylinder from the central borehole where the blast took place) that have propagated from the lower left corner (where the borehole is) and merged in the upper middle part of the image. This merging mechanism fragmented the corner material, i.e., just below the merging point, most likely due to the brittleness of the quartz grain located there. The characteristic structure of the branching-merging process leading to scale invariance is clearly visible in this figure: A large number of very small fragments is generated close to the main cracks, whereas larger but fewer fragments are generated further from the main cracks until the crack branches vanish at a characteristic cutoff size.

Figure 3(b) shows a region of a main radial crack propagated from the lower left (borehole) to the upper right corner of the image. The crack path, in this case, has fairly few microcracks compared to Fig. 3(a). However, the middle part of the crack includes branching cracks that follow grain boundaries that separate out some fragments. This illustrates how granularity influence fines formation.

\section{COMPUTATIONS}

\section{A. Numerical model}

We use a customized version of the HiDEM code [24] which has been developed for numerical simulation of brittle fracture. In HiDEM, the materials are discretized by in-elastic spheres that are connected by massless elastic Euler-Bernoulli beams. In the version used here, the discrete element model (DEM) particles are randomly packed using a dynamic deposition algorithm. The beams have square cross-sections $w^{2} \sim D_{\text {particle }}^{2}$, length $l \sim 2 D_{\text {particle }}$, and Young's modulus $E=19.7 \mathrm{GPa}$. Due to the random packing, the number of beams connecting adjacent particles varies between 8 and 20 . To mimic the experimental materials, initially, $10 \%$ of the beams are randomly chosen to have their stiffness reduced to $10 \%$ of the value of an intact beam. 
(a)

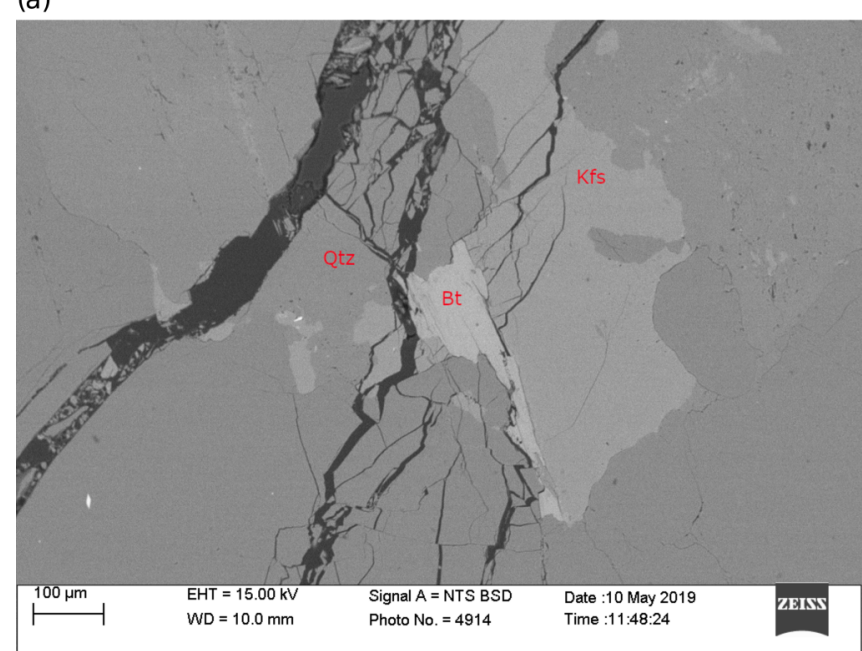

(b)

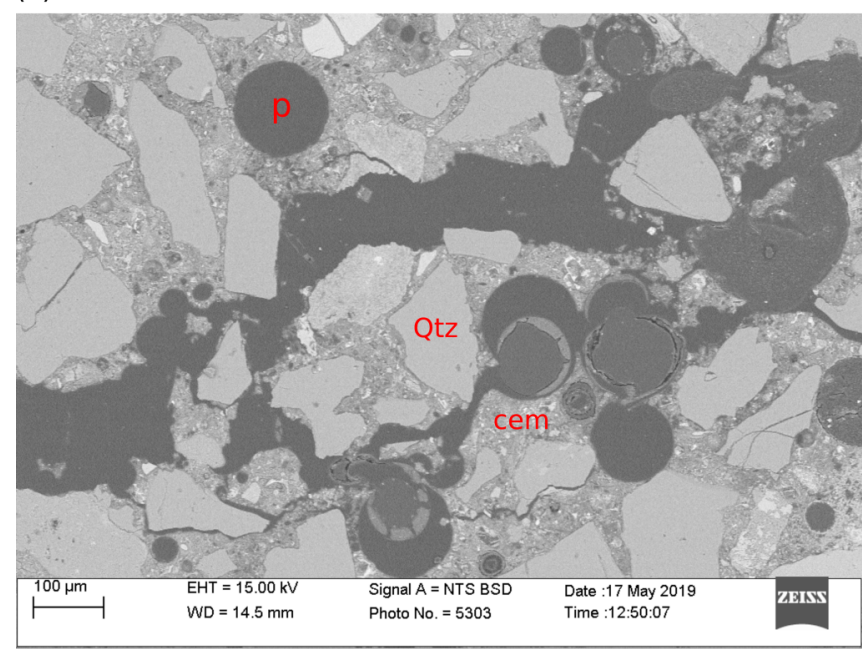

FIG. 3. SEM pictures of the branching and merging mechanism and fragment formation in (a) granite blasted with $20 \mathrm{~g} / \mathrm{m}$ of PETN (E2) with quartz (Qtz), biotite (Bt), and K-feldspar (Kfs) grains indicated on the image, and (b) mortar blasted with $12 \mathrm{~g} / \mathrm{m}$ of PETN (E1) with Qtz, cement (cem), and pores (p) indicated.

We have used a fracture criterion composed of two parts: (a) maximum strain criterion $\left(L-L_{0}\right) / L_{0}$, where $L_{0}$ is the original length of a beam, and $L$ the length of a stretched or compressed beam, and (b) a pure bending part $\operatorname{ABS}\left(\theta_{1}-\theta_{2}\right)$, where $\theta_{1}$ and $\theta_{2}$ are rotations of the end points of a beam. A beam breaks if deformation exceeds a threshold value for $(L-$ $\left.L_{0}\right) / L_{0}+$ const $\times \operatorname{ABS}\left(\theta_{1}-\theta_{2}\right)$. The threshold value used is $\varepsilon=0.0003$, i.e., typical tensile failure strain of mortar or concrete. We have tested also several other criteria including one in which we replace the rotation term with a shear term, however, the fragmentation results with this criterion match the experimental results best.

We do not have the computational resources to simulate the fragmentation of the full cylinders used in the experiments at high resolutions. With a grain size on the order of $0.1 \mathrm{~mm}$ and cylinders of size $\varnothing 140 \mathrm{~mm} \times 280 \mathrm{~mm}$ they would contain on the order of $10^{9}$ particles. Instead, to capture the dynamic fragmentation process along propagating cracks, we use the correct $0.1 \mathrm{~mm}$ resolution but compute fragmentation of

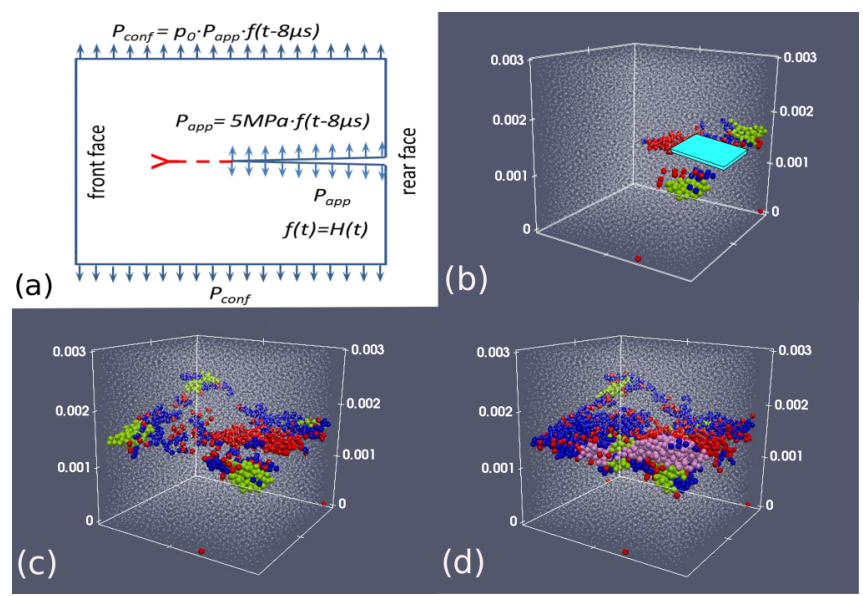

FIG. 4. (a) Schematic of the cube with a predefined crack, $H(t)$ is the Heaviside function. (b)-(d) show the evolution of the fragmentation of the cube $\left(3 \times 3 \times 3 \mathrm{~mm}^{3}\right)$ when $p_{0}=0$ at $t=2,4$, and $8 \mu \mathrm{s}$, respectively. In (b), the initial crack plane $\left(1.5 \times 1 \mathrm{~mm}^{2}\right)$ is shown in light blue. The cube's particles are shown with white points to improve the visibility of the fragments. Red: single particle fragments; blue: fragments containing two particles; green: fragments containing three to ten particles; pink: fragments containing 11 to 100 particles. (d) Represents the final stage of crack growth at $t=8 \mu \mathrm{s}$.

small scale cubes. We use cubes of size $3 \times 3 \times 3 \mathrm{~mm}^{3}$ with particles of diameter 0.1 and $6 \times 6 \times 6 \mathrm{~mm}^{3}$ with particles of diameter $0.05,0.08,0.1$, and $0.2 \mathrm{~mm}$. We initiate cracks with a predefined midplane edge crack. The initial crack is horizontal and goes half way through the cube. To mimic the blasting process, a dynamic pressure is applied on the initial crack flanks with a value $P_{\text {app }}=5 \mathrm{MPa}$ for a duration of $8 \mu \mathrm{s}$. To model the boundary conditions in the experiments, there is an additional external stress $P_{\text {conf }}=p_{0} \times P_{\text {app }}$ with $p_{0}$ as a constant that is applied on the top and bottom faces of the cube acting perpendicular to the crack flanks, see Fig. 4(a). As a representative example Figs. 4(b)-4(d) illustrate the time evolution of the dynamic crack propagation and the fragmentation in the small cube for an unstressed condition $P_{\text {conf }}=0 \mathrm{MPa}$. The sample breaks into a large number of small fragments, each composed of single or a few particles along the fracture plane due to the unstable tensile crack propagation induced by $P_{\text {app }}$, whereas the rest of the sample remains intact. The degree of fine-fragments generation depends on the ratio $p_{0}=P_{\text {conf }} / P_{\text {app }}$ that may be both negative and positive for compression and tension, respectively.

We performed fragmentation computations of the smaller cube with external stresses in the range of $P_{\text {conf }}=-0.2 P_{\text {app }}$ (compression) to $+0.15 P_{\text {app }}$ (tension), i.e., $p_{0}=-0.2$ to +0.15 in steps of 0.05 plus $p_{0}=+0.07$, altogether nine values. This models the dynamics of a nucleated crack propagating in a heterogeneous medium with different local crack tip region stresses, which occurs during the dynamic crack propagation of blast-induced cracks [25]. To average over several packing patterns, five different densely packed cubes were generated and fragmented for each external confinement pressure, i.e., external stresses, and their FSDs were added to each other to form a single FSD for each level of $P_{\text {conf }}$. We 
(a)

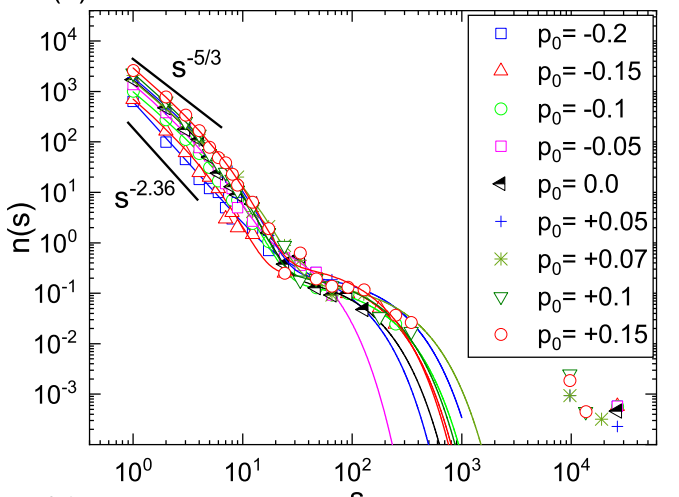

(c)

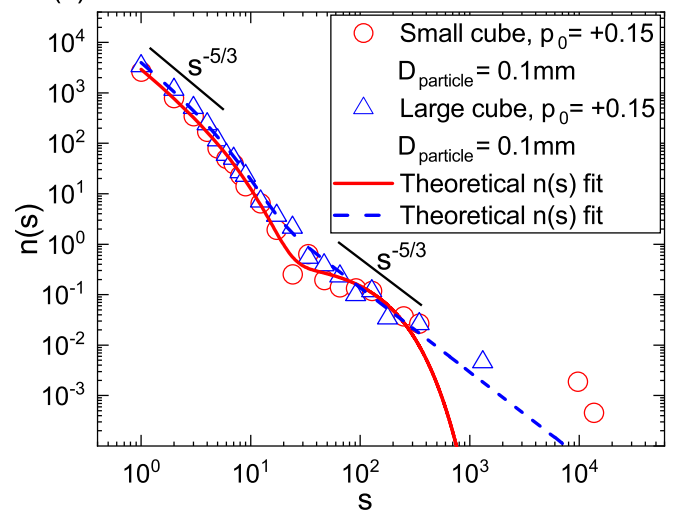

(b)
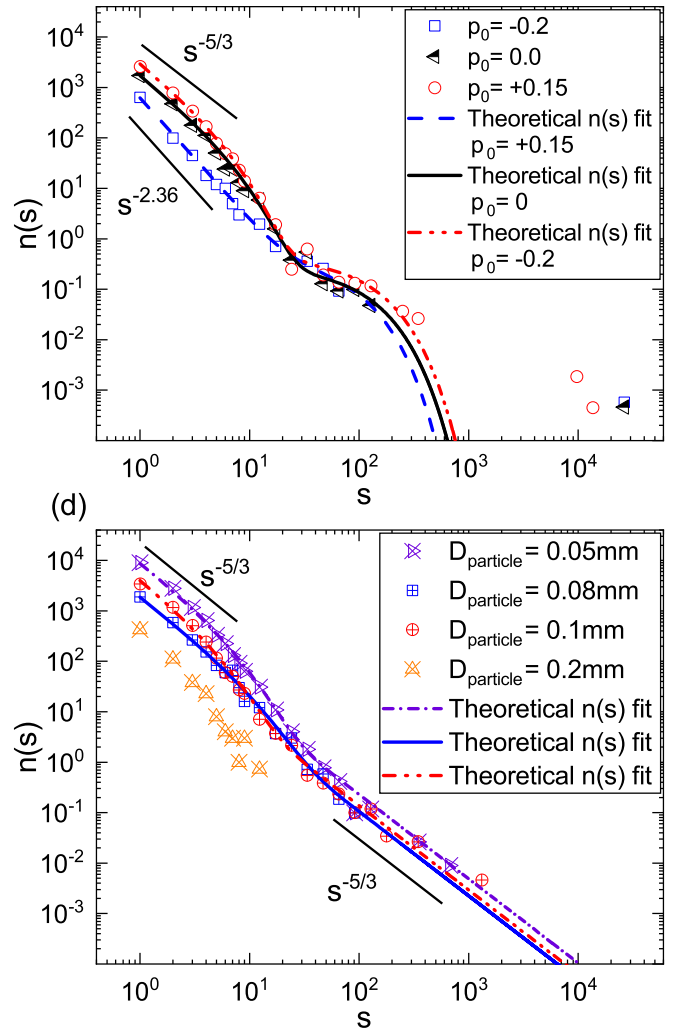

FIG. 5. (a) FSDs for dynamic crack propagation in the small cubes $\left(3 \times 3 \times 3 \mathrm{~mm}^{3}\right)$ for the nine different external stress levels and their $n(s)$ fits shown by the lines with the same color as the symbols. (b) FSDs for the unstressed and the two extreme external stress conditions with $p_{0}=+0.15$ and $p_{0}=-0.2$. Two lines with slopes of -2.36 and $-5 / 3$ are shown for comparison. (c) FSD for the large cube $\left(6 \times 6 \times 6 \mathrm{~mm}^{3}\right)$ with particle size $0.1 \mathrm{~mm}$ at $p_{0}=+0.15$ compared with that of the small cube at the same external stress condition. (d) FSDs for the large cube with four different particle sizes $0.2,0.1,0.08$, and $0.05 \mathrm{~mm}$ at $p_{0}=+0.15$. The corresponding theoretical $n(s)$ fits are shown by the lines. The lines with slopes of $-5 / 3$ are shown for comparison.

also computed FSDs for four larger cubes $\left(6 \times 6 \times 6 \mathrm{~mm}^{3}\right)$ with varying resolution (particle size) and at $p_{0}=+0.15$ to investigate the influence of the characteristic size of the model cube, the DEM-particle size, and the influence of boundary fragmentation [11].

\section{B. Numerical results}

Figure 5(a) shows the simulation results for the FSDs obtained with the nine external stress levels described above. In the figure, the theoretical $n(s)$ 's are fitted to the data using Eqs. (1) and (2) with the second term on the right-hand side neglected. The fine-fragments regions of the FSDs behave in the same way as in the experiments: The fine-fragments power-law exponent is close to $-5 / 3$ for positive $p_{0}$ and decreases to approximately -2.36 for the smallest $p_{0}$. The exponential cutoff for the fines appear at $s_{f} \sim 4-15$. The larger fragments can either be described by an exponential or there are just a few separate very large boulders.

In the simulations with the three highest external tensile stresses, the fracture plane completely splits the cube, creating two or three large boulders at $t \approx 7 \mu \mathrm{s}$. However, for the other stress levels, a large number of fine fragments is generated, but the cubes retained their integrity as the single largest fragment.

Figure 5(b) shows only FSDs for $p_{0}=-0.2,0.0,0.15$ to highlight the change in the power exponent for the fines. Table IV lists the regression analysis results for the computed

TABLE IV. Regression data for the fine region of FSD for the cube with the nine levels of external stresses. The values after the \pm sign are the standard error of the mean. Regression equation: $\ln \left(C_{1}\right)+\alpha \times \ln (s)-s / s_{f}$.

\begin{tabular}{|c|c|c|c|c|c|c|c|c|c|}
\hline Const. & -0.2 & -0.15 & -0.1 & -0.05 & 0 & +0.05 & +0.07 & +0.1 & +0.15 \\
\hline $\ln \left(C_{1}\right)$ & $6.32 \pm 0.15$ & $6.76 \pm 0.28$ & $7.10 \pm 0.18$ & $7.50 \pm 0.15$ & $7.68 \pm 0.12$ & $7.68 \pm 0.20$ & $7.78 \pm 0.14$ & $8.00 \pm 0.087$ & $8.14 \pm 0.082$ \\
\hline$\alpha$ & $-2.357 \pm 0.082$ & $-2.328 \pm 0.444$ & $-1.969 \pm 0.227$ & $-1.657 \pm 0.245$ & $-1.694 \pm 0.263$ & $-1.881 \pm 0.250$ & $-1.782 \pm 0.220$ & $-1.702 \pm 0.185$ & $-1.524 \pm 0.175$ \\
\hline$s_{f}$ & $2.62 \times 10^{30} \pm 0.0$ & $14.87 \pm 21.60$ & $11.10 \pm 4.83$ & $4.84 \pm 1.26$ & $5.07 \pm 1.78$ & $8.95 \pm 3.46$ & $7.12 \pm 2.44$ & $4.80 \pm 1.13$ & $4.24 \pm 0.83$ \\
\hline Adjusted $R^{2}$ & 0.9893 & 0.9687 & 0.9862 & 0.9899 & 0.9918 & 0.9837 & 0.9907 & 0.9961 & 0.9963 \\
\hline
\end{tabular}


TABLE V. Statistical significance study of the fine- and intermediate-size fragments' fitted lines for simulation of the large cube $\left(6 \times 6 \times 6 \mathrm{~mm}^{3}\right)$ with $D_{\text {particle }}=0.1 \mathrm{~mm}$.

\begin{tabular}{lcc}
\hline \hline Test & $\begin{array}{c}\text { Fine-fragments } \\
\text { region }\end{array}$ & $\begin{array}{c}\text { Intermediate-fragment- } \\
\text { size region }\end{array}$ \\
\hline No. data points & 3 & 10 \\
$R$ & -0.997 & -0.980 \\
$p(R)$ & $4.90 \times 10^{-2}$ & $7.16 \times 10^{-7}$ \\
Residual variance & $F=15.047$ & $p(F)=0.197$ \\
Gradient & $F=0.009$ & $p(F)=0.928$ \\
Intercept & $F=21.961$ & $p(F)=8.500 \times 10^{-4}$ \\
\hline \hline
\end{tabular}

data where the fine-fragments region of these FSDs are fitted with regression line $\ln \left(C_{1}\right)+\alpha \times \ln (s)-s / s_{f}$. The average value for the exponents for $p_{0} \geqslant-0.05$ in a total of six levels of external stresses is $-1.707 \pm 0.0490$. A critical compressive external stress is reached at $p_{0} \approx-0.1$ at which crushing becomes dominant over tensile fracture.

Since, the power-law range for the fines in Fig. 5(a) is extremely short, and the exponential cutoff begins to influence the exponent value, we also tested fitting the exponent using weights $1 / \sqrt{\ln }(s)$ in the fitting algorithm. Then, the average value of $-\alpha$ for $p_{0} \geqslant-0.05$ becomes, e.g., $-1.688 \pm 0.053$, which is even closer to $-5 / 3$.

The reason the power-law range for the fines is so small in the simulations compared to the experiments is that the smallest possible fragment in the simulations is a single grain. In the experiments, fragments get several orders of magnitude smaller. Note, however, that $s_{f}$ corresponds to fragments of linear size roughly in the range of (4-20) $\times x_{\text {grain }}$ for both experiments and simulations, indicating that it is, indeed, the grain size that determines the crossover between finesand intermediate-size-fragments regions. Note that $D_{\text {particle }} \sim$ $x_{\text {grain }}$.

Another discrepancy between experiments and simulations with the small cubes is the lack of the intermediate-size range in the simulated FSDs. The reason for this seems to be that almost all fragments larger than the fines are boundary fragments which tend to have an exponential FSD [11].

In order to demonstrate both of the above, we computed FSDs for larger cubes and for different $D_{\text {particle }}$ 's. The FSDs for a larger cube $\left(6 \times 6 \times 6 \mathrm{~mm}^{3}\right)$ with the same particle size of $x_{\text {grain }}=D_{\text {particle }}=0.1 \mathrm{~mm}$ at external stress level $p_{0}=$ +0.15 is compared to its counterpart for the smaller cube in Fig. 5(c). Table V lists the result of a significance study for the two fitted lines of fines- and intermediate-size fragments for the larger cube. The conclusion is the same as for the experiments: The power exponent of the FSD for the finesand the intermediate-size fragments are the same, and there is an offset between them.

Figure 5(d) shows FSDs for the larger cube $(6 \times 6 \times$ $6 \mathrm{~mm}^{3}$ ) with the four different particle sizes of $0.2,0.1$, 0.08 , and $0.05 \mathrm{~mm}$. The fine-fragment regions of the four simulations extend to roughly the same $s_{f} \sim 10$. Note that this means a 64 times smaller fragment volume for the smallest particles compared to the largest. This demonstrates that the fragment volume at the crossover between fines- and intermediate-size scales as the grain size $D_{\text {particle }}^{3}$.

\section{SUMMARY AND CONCLUSION}

Here, we report 28 experimental FSDs where four are confined with prestressed aggregate, and the rest are either unconfined or confined using a momentum trap. The fine fragments for six of them are sieved apart from the standard mechanical sieving with the sedimentation method down to $0.002 \mathrm{~mm}$. For an additional seven of them, the fine fragments are estimated with laser diffraction with a resolution down to $0.4 \mu \mathrm{m}$. These experimental FSDs, except the four that are confined with prestressed aggregates, have two fragment-size regions in which branching and merging of cracks generate a FSD of the universal scale-invariant form. These are the fine- and the intermediate-size fragment regions. Both can be described with a power-law exponent close to $-5 / 3$ with a statistically significant offset between them. If the blasted material is under compression induced by the prestressed confinement, the fine fragments are described by a steeper power law.

Numerical simulations of the same effect can be achieved using a detailed model of a pressurized crack propagating within a cube with randomly packed spherical elements and a midplane crack. Again, the FSD has two regions with an approximate exponent of $-5 / 3$ unless the crack is under strong enough external compressive stresses in which case the fines region has a steeper power law.

In both cases, the conclusions are supported by a statistical significance analysis. By varying the resolution in the numerical model, i.e., reducing the particle size, it is shown that the crossover between the two regions scales as the average grain size.

Within mining, it is common that operations require both blasting and ensuing crushing-grinding. The latter may well be the major contributor to the fines generation [4]. If the blast fragmentation is made coarser to reduce fines production by lowering the charge concentration, the need for increased crushing-grinding may quite possibly result in more total fine material from the operation. This makes the optimization of the process very challenging.

\section{ACKNOWLEDGMENTS}

This work has been funded by Austrian Science Fund (FWF): Project No. P27594-N29. We thank Prof. T. Antretter (Montanuniversität Leoben) for his valuable support.

\section{APPENDIX: EXPERIMENTAL MPFs}

The transformation from experimental $x-\operatorname{MPF}(x)$ data to $s-n(s)$ data uses the derivative of Eq. (3). Let $x_{\min }$ denote the smallest mesh then with $s=\left(x / x_{\min }\right)^{3}$,

$$
n(s)=C \frac{\operatorname{MPF}^{\prime}(x)}{s d s / d x}=C \frac{x_{\min }^{3} \operatorname{MPF}^{\prime}(x)}{3 x^{2} s(x)},
$$

with the prefactor $C=\frac{M_{0} x_{\min }^{3}}{3 m_{\text {grain }}}$. The value of the prefactor in Eq. (A1) shifts the curve along the $y$ axis in $\ln (s)-\ln [n(s)]$ space but does not change its shape. In our calculations, 
the prefactor $C=1$ was used. The difference quotient for two successive mesh sizes $x(i+1)$ and $x(i)$ is used to calculate

$$
\operatorname{MPF}^{\prime}[x(j)] \cong \frac{\operatorname{MPF}[x(i+1)]-\operatorname{MPF}[x(i)]}{x(i+1)-x(i)},
$$

at $x(j)=\sqrt{[x(i+1) x(i)]}$, the geometric mean (mid) points of all bins except the first $j=2, \ldots, n-1$. Two points are added to this shifted data set; $x(j=1)=x_{\min } / 2$ and $x(j=0)=0$ for which $\operatorname{MPF}(0)=0$. The equation $s(j)=$ $\left[x(j) / x_{\min }\right]^{3}$ defines the corresponding $s$ values, and Eqs. (A1) and (A2) define the corresponding $n[s(j)]$ values.
[1] J. S. Rinehart, Stress Transients in Solids (HyperDynamicS, New Mexico, 1975).

[2] D. Thornton, S. S. Kanchibotla, and I. Brunton, in Proceedings of EXPLO 2001 Conference, Hunter Valley, New South Wales, Australia, 2001, edited by A. Marton, (AusIMM, Carlton Victoria, Australia, 2001), p. 197.

[3] N. Djordjevic, Proceedings of 6th International Symposium for Rock Fragmentation by Blasting, Johannesburg, South Africa, 1999 (SAIMM, Johannesburg, South Africa), p. 213.

[4] I. Onederra, S. Esen, and A. Jankovic, Estimation of fines generated by blasting - applications for the mining and quarrying industries, Trans. Inst. Min. Metall., Sect. A 113, 237 (2004).

[5] A. Iravani, I. Kukolj, F. Ouchterlony, T. Antretter, and J. Åström, in Proceedings of 12th International Symposium for Rock Fragmentation by Blasting, Luleå, Sweden, 2018, edited by H. Schunnesson and D. Johansson (Luleå University of Technology, Luleå, Sweden, 2018), p. 597.

[6] D. Johansson, Fragmentation and waste rock compaction in small-scale confined blasting, Licentiate thesis, Luleå University of Technology, 2008.

[7] J. A. Åström, Statistical models of brittle fragmentation, Adv. Phys. 55, 247 (2006).

[8] A. Iravani, J. A. Åström, and F. Ouchterlony, Physical Origin of the Fine-Particle Problem in Blasting Fragmentation, Phys. Rev. Appl. 10, 034001 (2018).

[9] A. Meibom and I. Balslev, Composite Power Laws in Shock Fragmentation, Phys. Rev. Lett. 76, 2492 (1996).

[10] F. Wittel, F. Kun, H. J. Herrmann, and B. H. Kröplin, Fragmentation of Shells, Phys. Rev. Lett. 93, 035504 (2004).

[11] P. Gergő, I. Varga, and F. Kun, Emergence of energy dependence in the fragmentation of heterogeneous materials, Phys. Rev. E 90, 062811 (2014).

[12] E. Sharon and J. Fineberg, Microbranching instability and the dynamic fracture of brittle materials, Phys. Rev. B 54, 7128 (1996)

[13] R. Mahmoodi Baram, H. J. Herrmann, and N. Rivier, SpaceFilling Bearings in Three Dimensions, Phys. Rev. Lett. 92, 044301 (2004).
[14] J. A. Åström and J. Timonen, Spontaneous formation of densly packed shear bands of rotating fragments, Eur. Phys. J. E 35, 40 (2012).

[15] P. Kekäläinen, J. A. Åström, and J. Timonen, Solution for the fragmentation-size distribution in a crackbranching model of fragmentation, Phys. Rev. E 76, 026112 (2007).

[16] T. I. Riikilä, T. Tallinen, J. A. Åström, and J. Timonen, A discrete-element model for viscoelastic deformation and fracture of glacial ice, Comput. Phys. Commun. 195, 14 (2015).

[17] D. E. Grady and M. E. Kipp, Geometric statistics and dynamic fragmentation, J. Appl. Phys. 58, 1210 (1985).

[18] P. Moser, N. Cheimanoff, R. Ortiz, and R. Hochholdinger, in The First World Conference on Explosives and Blasting Technique, Rotterdam, 2000, edited by R. Holmberg (Balkema, Rotterdam, 2000), p. 165.

[19] B. A. Wills and J. Finch, Wills' Mineral Processing Technology: An Introduction to the Practical Aspects of Ore Treatment and Mineral Recovery (Butterworth-Heinemann, Oxford, 2015).

[20] H. C. Grimshaw, in Proceedings of Mechanical Properties of Non-Metallic Brittle Materials, London, 1958, edited by W. H. Walton (Butterworths, London, 1958), p. 380.

[21] C. Sun, Damage zone prediction for rock blasting, Ph.D. thesis, The University of Utah, 2013.

[22] I. Kukolj, A. Iravani, F. Ouchterlony, C. Weiss, and J. Lubensky, in Proceedings of 12th International Symposium for Rock Fragmentation by Blasting, Luleå, Sweden, 2018, edited by H. Schunnesson and D. Johansson (Luleå University of Technology, Luleå, Sweden, 2018), p. 483.

[23] T. J. Napier-Munn, Statistical Methods for Mineral EngineersHow to Design Experiments and Analyze Data (Julius Kruttschnitt Mineral Research Center, Queensland, Australia, 2014), Sec. 6.3.

[24] https://github.com/joeatodd/HiDEM.

[25] H. P. Rossmanith and K. Uenishi, On size and boundary effects in scaled model blasts spatial problems, Fragblast 9, 139 (2005). 